\title{
Quasi-parabolic pulses in a coherent nonlinear optical amplifier
}

\author{
Victor V. Kozlovi,2,* and Stefan Wabnitz ${ }^{2}$ \\ ${ }^{1}$ St.-Petersburg Univerisity, Department of Physics, Petrodvoretz, St.-Petersburg 198504 Russia \\ ${ }^{2}$ Dipartimento di Ingegneria dellInformazione, Universitá di Brescia, Via Branze 38, 25123 Brescia, Italy \\ ${ }^{*}$ Corresponding author: victor.kozlov@email.com
}

Received March 25, 2010; revised May 20, 2010; accepted May 21, 2010; posted May 25, 2010 (Doc. ID 126038); published June 10, 2010

\begin{abstract}
We show that, in a coherent two-level optical amplifier with Kerr nonlinearity and linear loss, any weak seed pulse evolves into a fixed powerful linearly chirped pulse with quasi-parabolic shape. This process is associated with a transition from the incoherent into the coherent amplification regime, thus enabling in practice the generation of pulses with a spectrum wider than the linear gain bandwidth. (c) 2010 Optical Society of America

OCIS codes: $140.4480,190.5530$.
\end{abstract}

Parabolic pulses are asymptotic self-similar solutions in the normal-dispersion regime of the amplified nonlinear Schrödinger equation (NLSE), which implies an ideal linear gain with indefinitely large bandwidth [1]. Such pulses, also known as similaritons, are characterized by a parabolic intensity profile and positive linear chirp [2]. In addition, the similariton peak power and its temporal and spectral width grow exponentially larger with distance. Propagation of parabolic pulses in amplifying normal-dispersion fibers followed by time compression by a grating pair results in the generation of 100 fs pulses with megawatt peak powers [3,4]. In practice, the finite linear gain bandwidth sets an upper limit to the spectral width of the generated pulses and, hence, a lower bound to the maximum temporal compression. In this Letter, we suggest an amplifier configuration where these limits are overcome.

The present scheme utilizes the nonlinear and coherent regime of propagation of pulses through a host Kerr medium doped with active two-level atoms/ions/quantum dots. In this case, the pulse spectral width is no longer limited by the spectral width of the gain but solely by the linear losses of the host medium [5], as in classical coherent nonlinear optical amplifiers. We analytically obtain an asymptotic stationary pulse solution (i.e., valid for large propagation distances) in the form of a central parabolic intensity shape with exponentially decaying wings and an approximately linear chirp. Numerical simulations show not only that such a solution is stable but also that it represents an attractor for the evolution of an arbitrary weak bell-shaped and initially unchirped seed pulse. Because of their steady-state rather than selfsimilar character, we name the present solutions quasiparabolic pulses.

The generic Maxwell-Bloch equations,

$$
\begin{gathered}
\left(\frac{\partial}{\partial z}+\frac{1}{c} \frac{\partial}{\partial t}\right) \Omega+l \Omega=g P+i f|\Omega|^{2} \Omega, \\
\frac{\partial}{\partial t} P=-\gamma_{\perp} P+\Omega N, \\
\frac{\partial}{\partial t} N=-\frac{1}{2}\left(\Omega P^{*}+\Omega^{*} P\right),
\end{gathered}
$$

supplemented by linear loss and Kerr-nonlinearity terms, describe the propagation of a slowly varying electric field $E(z, t)$ (represented by the Rabi frequency $\Omega=d E / \hbar$ where $d$ is the dipole moment of the two-level amplifier, and $\hbar$ the Planck constant). The above one-dimensional model applies to a waveguide, e.g., a doped optical microstructure fiber. The polarization $P(z, t)$ decays with rate $\gamma_{\perp}$, whereas the population difference $N(z, t)$ does not appreciably decay on the time scale of the pulse duration, so that the corresponding decay rate is set to zero. The coefficients $l>0, g>0$, and $f$ characterize linear fiber losses, small-signal gain of the active dopants, and Kerr nonlinearity, respectively.

We look for a solution in the form of a phasemodulated pulse with a stationary shape moving with the speed of light $c$, therefore, depending on the single variable $\tau=t-z / c$. By decomposing the field in amplitude $\alpha$ and phase $\phi$ as $\Omega=\alpha \exp (-i \phi)$, writing the polarization as $P=(p+i q) \exp (-i \phi)$ and by substituting into Eqs. (1) $-(\underline{3})$, we find

$$
\begin{gathered}
p=(l / g) \alpha, \\
q=-(f / g) \alpha^{3}, \\
N=N_{0}+(l / g) \int_{-\infty}^{\tau} \alpha^{2} \mathrm{~d} \tau^{\prime}, \\
\dot{\phi}=-\nu\left(3 \dot{\alpha} \alpha+\gamma_{\perp} \alpha^{2}\right) .
\end{gathered}
$$

Here, $N_{0}=N(\tau \rightarrow-\infty)=1, \nu=f / l$, and the dot denotes differentiation with respect to $\tau$. In terms of the new variable $F=\int_{-\infty}^{\tau} \alpha^{2} \mathrm{~d} \tau^{\prime}$, the equation for the field reads as

$$
\left(1+3 \nu^{2} \dot{F}^{2}\right) \ddot{F}+2\left(\gamma_{\perp} \nu^{2} \dot{F}^{2}+F-\tau_{p}^{-1}\right) \dot{F}=0,
$$

where $\tau_{p}^{-1}=(g / l) N_{0}-\gamma_{\perp}$ is the inverse pulse duration. Indeed, from the linearized version of Eq. (8) in the limit $\tau \rightarrow-\infty$, it follows that $\tau_{p}$ represents the pulse duration. In this limit, and after transforming $F$ back to $\alpha$, we get $\dot{\alpha}=\tau_{p}^{-1} \alpha$. So, $\tau_{p}$ is the inverse of the growth rate of the pulse front edge, which is the definition of pulse duration 
(not to be confused with FWHM). Equation (8) is quadratic in $\nu$; therefore, its solutions are insensitive to the sign of the Kerr nonlinearity.

In the limit of $\tau \rightarrow+\infty$, Eq. (8) determines the pulse energy $J=F(+\infty)$. Since $\dot{F} \rightarrow 0$, for $\tau \rightarrow+\infty$ for any localized pulse, we find $J=2 \tau_{p}^{-1}$ after integrating Eq. (8) once. Note that the energy $J$ remains the same for all values of $\nu$. In further analytical developments, we limit ourselves to the coherent limit, i.e., set $\gamma_{\perp} \rightarrow 0$. By integrating Eq. (8) once, we arrive to the cubic equation with respect to $F$ :

$$
\dot{F}+\nu^{2} \dot{F}^{3}=(J-F) F .
$$

This equation has one real and two complex roots. Only the real root,

$$
\dot{F}=\bar{f}(F) \equiv \sqrt{\frac{1}{3 \nu^{2}}}\left(u_{+}+u_{-}\right),
$$

with

$$
u_{ \pm}=\sqrt[3]{\eta \pm \sqrt{\eta^{2}+1}}
$$

and $\eta=\frac{1}{2} \sqrt{27 \nu^{2}}(J-F) F$, has physical meaning. In general, the integral

$$
\int_{0}^{F} \frac{\mathrm{d} F^{\prime}}{\overline{\bar{f}\left(F^{\prime}\right)}}=\int_{-\infty}^{t} \mathrm{~d} t^{\prime}
$$

and may be numerically evaluated, so that, from $F(\tau)$, we may recover the amplitude $\alpha(\tau)$. For $\nu=0$, the integral in Eq. (11) may be analytically evaluated and yields $\alpha(\tau)=$ $\tau_{p}^{-1} \operatorname{sech}\left(\tau / \tau_{p}\right)$. Quite general conclusions can be made about the behavior of the population difference at large times $(\tau \rightarrow \infty)$. From Eq. (6) and the expressions for the energy $J$ and the duration $\bar{\tau}_{p}$, it follows that $N(+\infty) \rightarrow-1$ for any $\nu$, that is, each stationary pulse of the family turns the amplifier into an absorber on the trailing edge of the pulse, similarly to classical $\pi$ pulses in a coherent amplifier.

Plots in Fig. 1 illustrate the basic properties of stationary quasi-parabolic pulses. The central part of the intensity profile is indeed parabolic as it is demonstrated by a parabolic fit, while the pulse wings decay exponentially to zero, as it follows from the analysis of Eq. (8). A direct
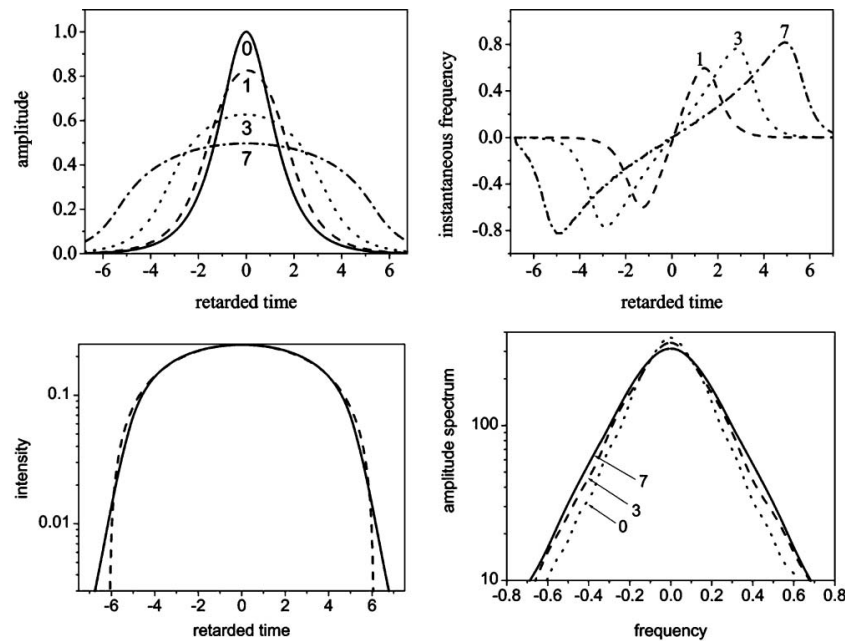

Fig. 1. (Top) Stationary amplitude $\alpha(t)$ and frequency $\dot{\phi}(t)$ profiles for four values of the Kerr nonlinearity: $\nu \tau_{p}^{-2}=0,1,3$, and 7, plotted as solutions of Eq. (11). Time is in units of $\tau_{p}$, Rabi frequency $\alpha$ and frequency $\dot{\phi}$ are in units of $\tau_{p}^{-1}$. (Bottom) Intensity profile $\left(\alpha^{2}\right)$ for $\nu=7 \tau_{p}^{2}$ (solid curves), fitted by a parabola (dotted curves), and corresponding spectra for $\nu \tau_{p}^{-2}=0,3$, and 7. Here $\gamma_{\perp}=0$.

consequence of the parabolic intensity shape is the linear increase of the frequency chirp, which is observed in central portion of the pulse. Note that the spectral bandwidth of the pulses remains approximately constant for all $\nu$. It is such a bandwidth that sets a lower limit to pulse shortening by an appropriate compressor. We conclude that the quasi-parabolic pulses cannot be compressed any further than the non-phase-modulated pulses of a hyperbolic secant shape that are generated by the same amplifier in the absence of Kerr nonlinearity. With increasing $\nu$, the quasi-parabolic pulses widen in time; given that, for a fixed ratio $l / g$, their energy remains the same, the pulse amplitude correspondingly decreases.

Figure 2 (for $\gamma_{\perp}=0$ ) and Fig. 3 (for $\gamma_{\perp} \neq 0$ ) show the evolution of a weak seed pulse toward a stationary quasiparabolic pulse with linear (positive for $\nu>0$ and negative for $\nu<0$ ) chirp, which demonstrates the stability of our solutions. Figure 3 shows the transition from the regime of incoherent interaction (i.e., with $\gamma_{\perp} \tau_{0}>1$ ) into
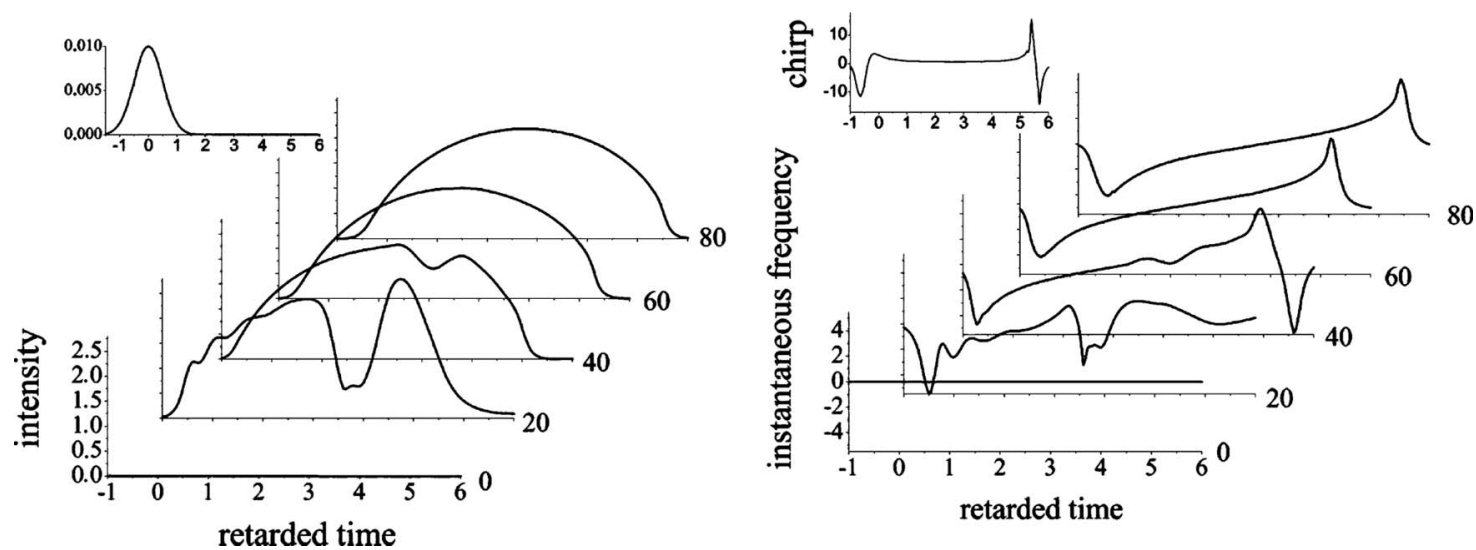

Fig. 2. Evolution of initial non-phase-modulated Gaussian pulse of duration $\tau_{0}$ for $\nu=3 \tau_{0}^{2}, l=0.2$ g, and $\gamma_{\perp}=0$ toward a stationary shape. The seed pulse is not resolved on the main figure, and it is shown in the left inset. Amplitude, frequency, and time are normalized to $\tau_{0}$. Numbers on the right show the distance measured in units of the resonant length $\left(g \tau_{0}\right)^{-1}$. 

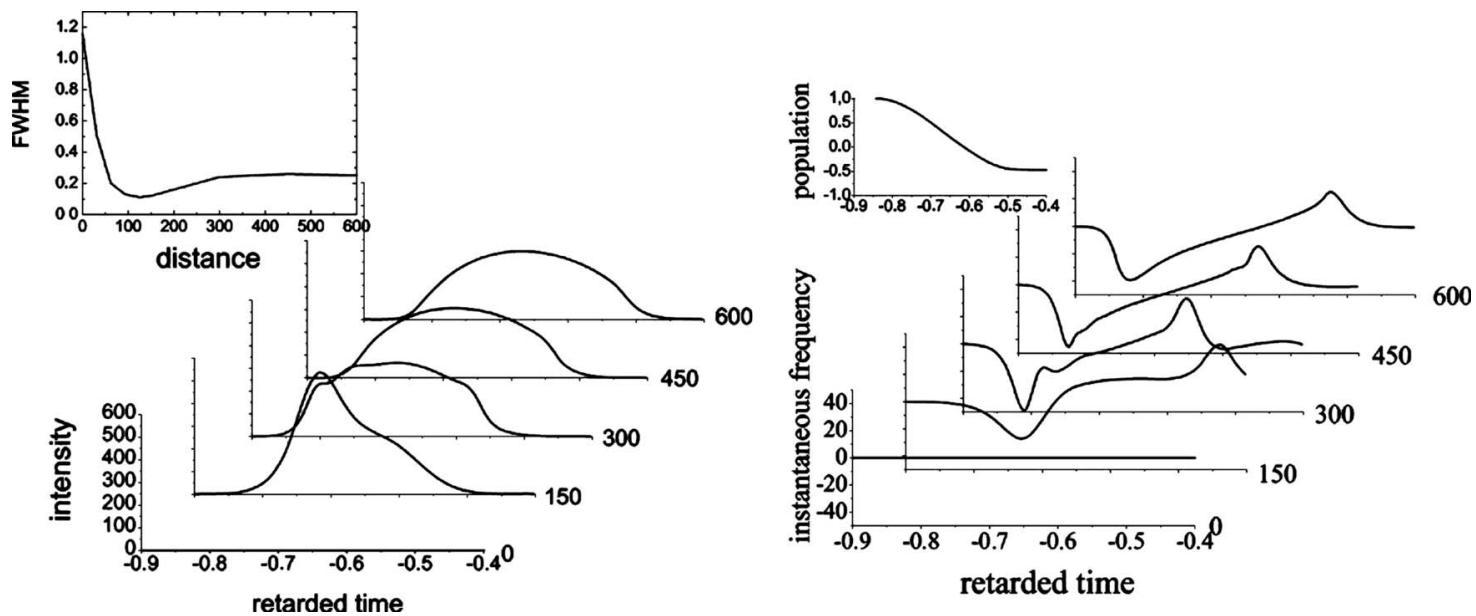

Fig. 3. Same as in Fig. 2 for $\gamma_{\perp} \tau_{0}=4$ with the same seed pulse, demonstrating the dynamic breaking of the linear gain bandwidth barrier $\left(\tau_{p} \gamma_{\perp}=1\right)$, i.e., evolution of the temporal FWHM (shown in the left inset) from $\tau_{0}>\gamma_{\perp}^{-1}$ to $\tau_{p}<\gamma_{\perp}^{-1}$. The right inset shows the output inversion profile. Here $l=0.02 \mathrm{~g}$ and $\nu=0.0125 \tau_{0}^{2}$.

the coherent regime (i.e., with $\gamma_{\perp} \tau_{p}<1$ ). This transition is accompanied by fivefold shortening of the pulse temporal FWHM, and it leads to breaking the limitation $\tau_{p}>\gamma_{\perp}^{-1}$, which is set to the pulse duration $\tau_{p}$ by the linear gain bandwidth. Additional temporal compression can be achieved by compensating the linear chirp of the quasiparabolic pulse by an appropriate compressor. The onset of a coherent interaction is marked by the development of characteristic negative regions in the inversion profile. On the pulse trailing edge, the inversion does not reach its absolute minimum equal to -1 , as it occurs for coherent $\pi$ pulses, because the emerging quasi-parabolic pulse is not yet short enough when compared with $\gamma_{\perp}^{-1}$.

In linear amplifiers, in the absence of the abovedescribed nonlinear pulse compression mechanism, the lower limit to the pulse duration is fundamentally determined by the finite linear gain bandwidth. On the other hand, we have shown that breaking through this limit may be obtained, thanks to the power broadening of the two-level amplifier by the strong pulse. For instance, rare-earth-doped fibers behave as linear amplifiers for the usual megawatt range of peak powers of amplified picosecond and femtosecond pulses. For entering the nonlinear and coherent amplification regime, gigawatt peak powers would be necessary, which almost certainly would damage the material. In contrast, with a microstructure fiber doped with quantum dots exhibiting gigantic dipole moments [6], a peak power of a few tens of watts in a pulse of less than $100 \mathrm{fs}$ pulse would be sufficient to implement the nonlinear and coherent regime of interaction. In addition, the present study is practically relevant to the case of semiconductor optical amplifiers.

The quasi-parabolic pulses are shaped by two physical mechanisms. The first and dominant mechanism forms stationary resonant coherent $\pi$ pulses through a balance between coherent nonlinear gain responsible for temporal compression and linear broadband losses. The second shaping mechanism results from a counterbalance be- tween spectral broadening induced by the Kerr nonlinearity and the resonant dispersion of the amplifying two-level systems. Such dispersion is induced by the pulse itself and is purely nonlinear; see $q$ in Eq. (5). Linear resonant dispersion is strictly equal to zero. Therefore, the second mechanism is basically different from the physics of soliton formation in the NLSE. Indeed, it is remarkable that the pulse leads to anomalous nonlinear resonant dispersion for a positive sign of the Kerr nonlinearity and to normal dispersion for a negative Kerr nonlinearity. For either sign of $f$, dispersion counterbalances the Kerr nonlinearity for arbitrary large values of $f$, in contrast with the case of two-level absorbers, where the formation of stationary shapes is possible only for $f$ smaller than a threshold value [7].

In conclusion, a two-level coherent nonlinear amplifier with Kerr nonlinearity is shown to support the formation of stationary quasi-parabolic $\pi$ pulses with linear chirp. This chirp allows for a compression of the emerging quasi-parabolic pulse by a pair of diffraction gratings. The nonlinearity of the amplifier allows for breaking the limit on the pulse shortening, which is set by the finite linear gain bandwidth.

\section{References}

1. K. Tamura and M. Nakazawa, Opt. Lett. 21, 68 (1996).

2. M. E. Fermann, V. I. Kruglov, B. C. Thomsen, J. M. Dudley, and J. D. Harvey, Phys. Rev. Lett. 84, 6010 (2000).

3. T. Schreiber, C. K. Nielsen, B. Ortac, J. Limpert, and A. Tünnermann, Opt. Lett. 31, 574 (2006).

4. D. N. Papadopoulos, Y. Zaouter, M. Hanna, F. Druon, E. Mottay, E. Cormier, and P. Georges, Opt. Lett. 32, 2520 (2007).

5. J. P. Wittke and P. J. Warter, J. Appl. Phys. 35, 1668 (1964).

6. H. C. Y. Yu, A. Argyros, G. Barton, M. A. van Eijkelenborg, C. Barbe, K. Finnie, L. Kong, F. Ladouceur, and S. McNiven, Opt. Express 15, 9989 (2007).

7. V. V. Kozlov and É. E. Fradkin, Opt. Lett. 20, 2165 (1995). 